\title{
Artesunate prevents knee intraarticular adhesion via PRKR-like ER kinase (PERK) signal pathway
}

\author{
Hui Chen ${ }^{1+}$, Jin Tao ${ }^{2+}$, Jingcheng Wang ${ }^{1,2^{*}}$ and Lianqi Yan ${ }^{1,2^{*}}$
}

\begin{abstract}
Background: Intraarticular scar adhesion refers to a serious complication caused by knee surgery or trauma, leading to various sequelae (e.g., articular cartilage degeneration and knee joint stiffness). Artesunate (ART) has exhibited an effect to suppress fibroblast proliferation, whereas the exact mechanism remains unclear. This study aims to delve into the possible mechanism of ART in suppressing joint adhesion.

Methods: The effect of ART on reduced intraarticular adhesions was ascertained by histological staining and immunohistochemical analysis through vivo experiments. Cell Counting Kit-8 (CCK-8) assay, Western blot analysis, flow cytometry, and tunnel staining were used to detect the effect of ART in promoting fibroblast apoptosis and delve into its possible signaling pathway.

Results: The results of hematoxylin-eosin (HE) staining suggested that the number of fibroblasts decreased with the increase in ART concentration. The results of Masson staining were similar, with the increase in concentration, the collagen content decreased. Immunohistochemical results showed that the expression of endoplasmic reticulum stress (ERS) characteristic proteins $78 \mathrm{kDa}$ glucose-regulated protein 78 (GRP78) and C/EBP homologous protein (CHOP) increased in a concentration-dependent manner. CCK-8 results suggested that ART could inhibit fibroblast viability in a concentration- and time-dependent manner. Results of flow cytometry, tunnel staining, and Western blot suggested the apoptosis of fibroblasts occurred after ART treatment. Cells with caspase inhibitors were treated, and apoptotic proteins cleaved-poly ADP-ribose polymerase (cleaved PARP) and cleaved-caspase 3 were detected; the results showed that the apoptotic effect of ART was reduced. The expressions of ERS-related protein CHOP and apoptosis-related protein Bax were upregulated, while the expression of $\mathrm{BCl}-2$ was downregulated, and the ratio of Bax/BCl-2 increased in a concentration-dependent manner. Continuous detection of PRKR-like ER kinase (PERK) pathway-related proteins showed that the expression of p-PERK and phosphorylating eukaryotic initiation factor $2 a$ ( $p$-elF2a) increased in a time-dependent and concentration-dependent manner. PERK pathway inhibitors could partially inhibit ART-mediated apoptosis through PERK pathway.
\end{abstract}

Conclusions: ART can promote fibroblast apoptosis through PERK pathway, a classical ERS pathway, and thus prevent fibrosis in the surgical area after joint surgery.

Keywords: Artesunate (ART), PRKR-like ER kinase (PERK), Apoptosis, Intraarticular scar adhesion

\footnotetext{
*Correspondence: jingchengwyz@163.com; yanlianqi@126.com

${ }^{+} \mathrm{Hui}$ Chen and Jin Tao contributed equally to this work.

'Dalian Medical University, Dalian, Liaoning, People's Republic of China

Full list of author information is available at the end of the article
}

(C) The Author(s). 2019 Open Access This article is distributed under the terms of the Creative Commons Attribution 4.0 International License (http://creativecommons.org/licenses/by/4.0/), which permits unrestricted use, distribution, and reproduction in any medium, provided you give appropriate credit to the original author(s) and the source, provide a link to the Creative Commons license, and indicate if changes were made. The Creative Commons Public Domain Dedication waiver (http://creativecommons.org/publicdomain/zero/1.0/) applies to the data made available in this article, unless otherwise stated. 


\section{Introduction}

The main weight-bearing joint, the knee joint, is prone to injury and degenerative lesions for its high mobility and lack of soft tissue protection. Intraarticular scar adhesion of knee joint refers to a common complication after surgery or trauma, and it critically affects the prognosis of surgery. It has been one of the difficult problems facing the world's orthopedic department. It often causes complications (e.g., the limited range of motion of joints, chronic joint pain, and even degenerative diseases of articular cartilage) [1]. Accordingly, a way is required to address this problem in the clinic.

Numerous ways have been developed to mitigate intraarticular adhesion after knee surgery or trauma. For instance, some scholars studied the efficacy of manual lysis in the treatment of knee joint adhesions under anesthesia. Their results suggested that this method exhibits significant advantages in the treatment of early knee joint adhesion [2]. Others also proposed to use drugs (e.g., the use of mechanical barrier drugs, antiinflammatory drugs, inhibition of fibroblasts drugs) to prevent knee adhesions and have achieved certain results in basic experiments [3-6]. However clinically, there is still controversy about the feasibility of drug-induced knee adhesions, so new drugs which have small side effects to solve this problem are required.

Artesunate (ART), i.e., artesunatum, is one of the artemisinin derivatives that exhibit a sesquiterpene structure. It is a fast-acting, low-toxic antimalarial drug used for the treatment of malaria parasite infection in adults, children, and pregnant women, having saved millions of lives $[7,8]$. Its biological activity and metabolites have been widely discussed, including anti-inflammatory, anti-sepsis, anti-tumor, radio-sensitization, antibacterialsensitization, etc. [9-12].

ART, an antimalarial drug, also exhibits certain antifibrotic effects (e.g., pulmonary fibrosis, liver fibrosis, and renal fibrosis) [13-15]. ART can significantly downregulate the expression of TGF- $\beta 1$ and TNF- $\alpha$ in rats with pulmonary fibrosis, thereby mitigating the pulmonary fibrosis. It also can prevent oxidative stress, suppress proinflammatory cytokines, inhibit hepatic stellate cell proliferation, and induce hepatic stellate apoptosis, thus affecting the synthesis and metabolism of extracellular matrix and exerting anti-hepatic fibrosis [16]. Accordingly, we assumed that ART can also suppress fibroblast proliferation, thereby mitigating postoperative adhesions in the knee joint.

The endoplasmic reticulum (ER) is a vital organelle involved in protein synthesis, modification and processing, as well as the folding, the assembly, and the transport of nascent peptide chains. The classical mechanisms of endoplasmic reticulum stress (ERS)-mediated apoptosis are primarily classified into two types (unfolded protein response and calcium signaling) [17]. On the one hand, once unfolded or misfolded proteins are assembled, the unfolded protein response (UPR) will serve as a protective mechanism to protect cells from being harmed. However, if the unfolded protein or the misfolded protein is not eliminated, it may cause apoptosis [18]. On the other hand, the calcium homeostasis of the endoplasmic reticulum of the cell is also crucial to the cell. Once the calcium homeostasis is broken, the apoptosis will be induced. In recent years, it has been reported that ERS-mediated apoptosis is conducive to the treatment of fibrotic diseases. PRKR-like ER kinase (PERK) suppresses protein synthesis in cells by phosphorylating eukaryotic initiation factor $2 \alpha$ (eIF2 $\alpha$ ), and it upregulates C/EBP homologous protein $(\mathrm{CHOP})$ gene by activating transcriptional activator 4 . The expression of activated apoptotic pathways is involved in the cellular stress response process, and the PERK pathway is one of the critical pathways for ERS. It has recently been reported that ERS-mediated apoptosis is capable of treating fibrotic diseases [19].

In the present study, we aimed to investigate whether ART can induce fibroblast apoptosis through PERK pathway, reduce, or even prevent intraarticular adhesion after knee surgery or trauma. We considered it an effective method to mitigate knee joint adhesion.

\section{Materials and methods}

This experimental study was approved by the Ethics Committee and Research Committee of Jiangsu Subei People's Hospital. Primary human fibroblast cell line was provided by GuangZhou Jenino Biotech Co., Ltd. Fibroblasts were cultured in Dulbecco's modified Eagle's medium (DMEM; Gibco, USA) containing 20\% fetal bovine serum (FBS; Gibco) and $100 \mathrm{U} / \mathrm{ml}$ penicillin and $100 \mathrm{U} / \mathrm{ml}$ streptomycin (Thermo USA). The cells were incubated in an incubator at $37{ }^{\circ} \mathrm{C}$ and $5 \% \mathrm{CO}_{2}$. When the fibroblasts grow to the 4th to 7th generations, the cells exponentially growing at this time will be used for cell experiments.

\section{ART treatment}

ART was provided by Li Shizhen Pharmaceutical Co., Ltd. (Hubei, China). Fibroblasts were cultured in 6-well plates, 96-well plates, or 10-cm-diameter culture dishes. GSK2606414, a PERK signaling pathway inhibitor, was purchased from Medchem Express Co., Ltd. (Shanghai, China). Q-VD-Oph, a caspase signaling pathway inhibitor, was purchased from Medchem Express Co., Ltd. (Shanghai, China). When the cells were grown to nearly $80 \%$, they were rinsed with phosphate buffer saline (PBS) for three times. Human fibroblasts were treated with ART. The control group was incubated with PBS. Subsequently, experimental and control cells were collected for subsequent cell experiments. 


\section{Cell viability assay}

The Cell Count Kit-8 (CCK-8, Dojindo, Tokyo, Japan) was employed for testing fibroblast viability. The fifth to seventh generations of fibroblasts were taken, and the cells were planted in two 96-well plates. In one group, when the cells are grown to about $80 \%$, the cells will be treated with ART at different concentrations $(0,5 \mu \mathrm{M}$, $10 \mu \mathrm{M}, 20 \mu \mathrm{M}, 40 \mu \mathrm{M}$, and $80 \mu \mathrm{M})$. Twenty-four hours later, the metabolites were first removed through PBS buffer washing, and then DMEM $100 \mu \mathrm{l}$ and $10 \mu \mathrm{l} \mathrm{CCK-}$ 8 were added to further culture the cells for $2 \mathrm{~h}$. In the other group, when grown to $80 \%$, the cells will be treated with $10 \mu \mathrm{M}$ for $0,12,24,36,48,60$, and $72 \mathrm{~h}$. Their optical densities were measured at $450 \mathrm{~nm}$ using a Microplate absorbance reader (Bio-Tek, Elx 800, USA). Cell viability was calculated based on the reference manual.

\section{Cell apoptosis was analyzed by flow cytometry}

Viable cells were collected and planted in 6-well plates, and then the cells were placed in the incubator overnight. Three well cells in a 6-well plate were classified as a control group, and the remaining were cultured for $12 \mathrm{~h}$ in the incubator by being treated with $10 \mu \mathrm{M}$. Then, the cells were harvested and rinsed three times with an appropriate amount of PBS buffer solution. To grow suspension cells, the cells were suspended in $1 \mathrm{ml}$ binding buffer $\times 1$, and $100 \mu \mathrm{l}$ of cell suspension was transferred to a test tube. Subsequently, $5 \mu$ l FITC Annexin $\mathrm{V}$ and $5 \mu \mathrm{l}$ PI were added to each tube. Finally, a $400 \mu \mathrm{l} 1 \times$ binding buffer was added to a test tube, and the cells were stored at ambient temperature in a light proof way. The mixture containing FITC Annexin V and PI was incubated for $15-20 \mathrm{~min}$, and the apoptosis of the mixture was finally detected by flow cytometry.

\section{Western blot analysis}

Different concentrations of ART treated cells and cells of the same concentration of ART were harvested for different treatment times to conduct Western blot analysis. First, the collected fibroblasts were treated on ice with a radioimmune-deposition (RIPA) buffer (Beyotime, Hangzhou, China). Subsequently, sonicate and centrifuge processing were performed to harvest proteins. Lastly, the concentration of protein was detected using BCA Protein Assay Kit (Beyotime, Hangzhou, China). Further, $40 \mu \mathrm{g}$ of each protein lysate were separated on the Western blot at a $5-12 \%$ sodium dodecyl sulphate-polyacrylamide gel electrophoresis (SDS-PAGE) and then transferred to polyvinylidene difluoride (PVDF) membranes (Millipore, Bedford, MA) for $1.5 \mathrm{~h}$ at $200 \mathrm{~mA}$. Next, the PDVF membrane was blocked for $2 \mathrm{~h}$ at ambient temperature with 5\% skimmed milk. Then, PVDF membrane was washed with tris-buffered saline and Tween20 (TBST) solution. Following the reference manual, PVDF membranes were incubated with primary and secondary antibodies continuously. The primary antibodies were used, including anti-cleaved-poly ADP-ribose polymerase (cleaved PARP), anti-cleaved caspase3, anti-Bax, anti-Bcl2, anti-78 $\mathrm{kDa}$ glucose-regulated protein (GRP78), anti-CHOP, anti-PERK, anti-phospho-PERK (p-PERK), anti-eukaryotic translation initiation factor $2 \alpha$ (eIF2 $\alpha$ ), anti-phospho-eIF2 $\alpha$ (p-eIF2 $\alpha$ ), and anti-glyceraldehyde-3-phosphate dehydrogenase $(\mathrm{GAPDH})$ and secondary antibodies including antimouse or antirabbit IgG. All of the above antibodies were purchased from Cell Signaling Technology (Beverly, MA, USA); the concentration of the antibodies used in this study was $1 \mu \mathrm{g} / \mathrm{ml}$.

\section{TUNEL assay in fibroblasts}

Tunnel staining was employed to test apoptosis of human fibroblasts (KeyGEN, Nanjing, China). The experimental methods rigorously followed the manufacturer's instructions. Fluorescence microscope was used to observe the features of apoptosis. Fibroblasts stained by TUNEL were considered apoptotic. The total number of fibroblasts was calculated using DAPI staining.

\section{Animals}

The animal experiment was approved by the Animal Ethics Committee of Yangzhou University, and all the rabbits received humanitarian care. Twenty-four mature 4-month-old male New Zealand rabbits weighed $3.8 \mathrm{~kg}$ for this study. Rabbits were split into four groups at random (six in each group), including ART (15 mg/kg) group, $(30 \mathrm{mg} / \mathrm{kg})$ group, $(60 \mathrm{mg} / \mathrm{kg})$ group, and control group. Before the experiment, rabbits were kept in captivity for 1 week to adapt to the laboratory environment.

\section{Local application of ART}

An intraarticular adhesion animal model was built based on previous scholars' studies [20]. New Zealand rabbits were first given an intravenous injection of $2 \%$ pentobarbital $(1.5 \mathrm{ml} / \mathrm{kg})$ to achieve general anesthesia. Subsequently, the hair around the knee joint was shaved, the exposed skin was disinfected with iodophor, and the femoral condyle was then exposed. The cortical bone of nearly $10 \mathrm{~mm} \times 10 \mathrm{~mm}$ was removed on both sides of the femoral condyle, and the articular cartilage was protected carefully. After the operation area was disinfected and hemostasis, the wound would be sutured. Next, the skin disinfection in the surgical area was achieved, and local injection concentrations of 15,30 , and $60 \mathrm{mg} / \mathrm{kg}$ ART were applied in the corresponding experimental group. The control group was injected with an equal amount of physiological saline. The drug was injected once per 2 days for five times. Furthermore, after the surgery, the cefazolin sodium was intramuscularly 
injected for 3 days to prevent postoperative infection of the rabbit; the surgical knee joint was externally fixed for 4 weeks in the Kirkner wire bending position.

\section{Histological analysis}

Histological analysis was conducted 4 weeks after surgery. One rabbit was taken from each group at random. Subsequently, the rabbits were euthanized, the knee joint was opened along the original incision, and then the knee joint was removed (including all connective tissue and fibrous adhesion scar). The samples were fixed in 10\% formalin for 1 week and then decalcified for 2 weeks. The tissue was embedded in paraffin, and transverse sections $5 \mathrm{~mm}$ perpendicular to the femoral axis were removed and stained with hematoxylin-eosin (HE) and Masson's trichrome, respectively. Intraarticular scar adhesion and intraarticular collagen tissue were further evaluated under a $\times 400 \mathrm{mag}$ nification of an optical microscope.

\section{Immunohistochemical staining}

The expressions of GRP78 $(10 \mu \mathrm{g} / \mathrm{ml})$ and $\mathrm{CHOP}$ $(10 \mu \mathrm{g} / \mathrm{ml})$ of the sections were detected using immunohistochemical staining. After deparaffinization and rehydration in gradient ethanol, the sodium citrate was added to the distilled water to pretreat the sections. After sealing with $10 \%$ goat serum at ambient temperature for $30 \mathrm{~min}$, these sections were incubated with anti-GRP78 antibody and anti-CHOP antibody in an environment of $4{ }^{\circ} \mathrm{C}$. They were then incubated at ambient temperature for $2 \mathrm{~h}$ and then analyzed with a coating tool to detect antibody binding. Subsequently, the sections were stained with hematoxylin. Finally, these sections could be observed under an optical microscope.

\section{Statistical analysis}

SPSS 19.0 statistical software was employed to analyze the data. The independent sample $t$ test was used in our experiment. All data were expressed as the mean \pm standard deviation (S.D) values. Experiments were repeated three times. Statistical significance was defined as $P<0.05$.

\section{Results}

All animals received care following the principles of Laboratory Animal Care of international recommendations. The experimental protocol was approved by the Animal Care and Research Committee of the Yangzhou University, China. There were no surface or deep infections and no complications from surgical incisions.

\section{Histological analysis of the effect of ART on the intraarticular scar adhesion}

Several important information can be acquired from HE staining images. In the experimental group, the scar tissues of $15 \mathrm{mg} / \mathrm{kg}$, $30 \mathrm{mg} / \mathrm{kg}$, and $60 \mathrm{mg} / \mathrm{kg}$ was less than those of the control group, and the $60 \mathrm{mg} / \mathrm{kg}$ group was still lower than the $15 \mathrm{mg} / \mathrm{kg}$ and $30 \mathrm{mg} / \mathrm{kg}$ group (Fig. 1a). The number of fibroblasts was measured by image pro plus, and it was found that the number of fibroblasts gradually decreased as the concentration of ART rose (Fig. 1b). Masson's trichrome staining image displayed that, compared with the control group, collagen density in the experimental group was significantly lower than the control group. The trend is similar to that of HE staining (Fig. 1c, d). Based on the above results, we can preliminarily conclude that local application of ART can reduce the scar adhesion in the joint.

\section{ART inhibits intraarticular adhesion through ERS}

In order to further explore the mechanism, we examined the expression of markers GRP78 and CHOP by immunohistochemical analysis. Immunohistochemical analysis and the results of quantitative analysis of immunohistochemistry showed that GRP78 and CHOP in the cytoplasm of fibroblasts in the ART-treated group were significantly higher than those in the control group which showed a concentration-dependent manner (Fig. 2a-d). We can conclude that ERS was involved in ART-mediated delayed intraarticular adhesion.

\section{Effect of ART on fibroblasts}

Fibroblasts treated with different concentrations of ART and cultured with the same concentration of ART for different time were detected by cck-8 method. It was found that ART could inhibit the viability of fibroblasts in a concentration-dependent and time-dependent manner (Fig. 3a, b). To determine whether ART affects cell viability through apoptosis, we use tunnel staining and flow cytometry, and the results showed that ART could induce the apoptosis of fibroblasts (Fig. 3c-f). In addition, cleaved-PARP were used as apoptosis marker proteins; Western blot further showed that ART can induce fibroblast apoptosis (Fig. 3g, h). We use Q-VD-OPh, a caspase signaling pathway inhibitor, which demonstrated the cytoprotective effect of caspase inhibitors by Western blot quantitative analysis (Fig. 3i, j). In summary, ART can reduce cell viability through apoptosis.

\section{ART can induce fibroblast apoptosis through PERK pathway}

After different concentrations of ART treatment, it was found that the proapoptotic proteins $\mathrm{CHOP}$ and Bax were upregulated in expression, while the anti-apoptotic 


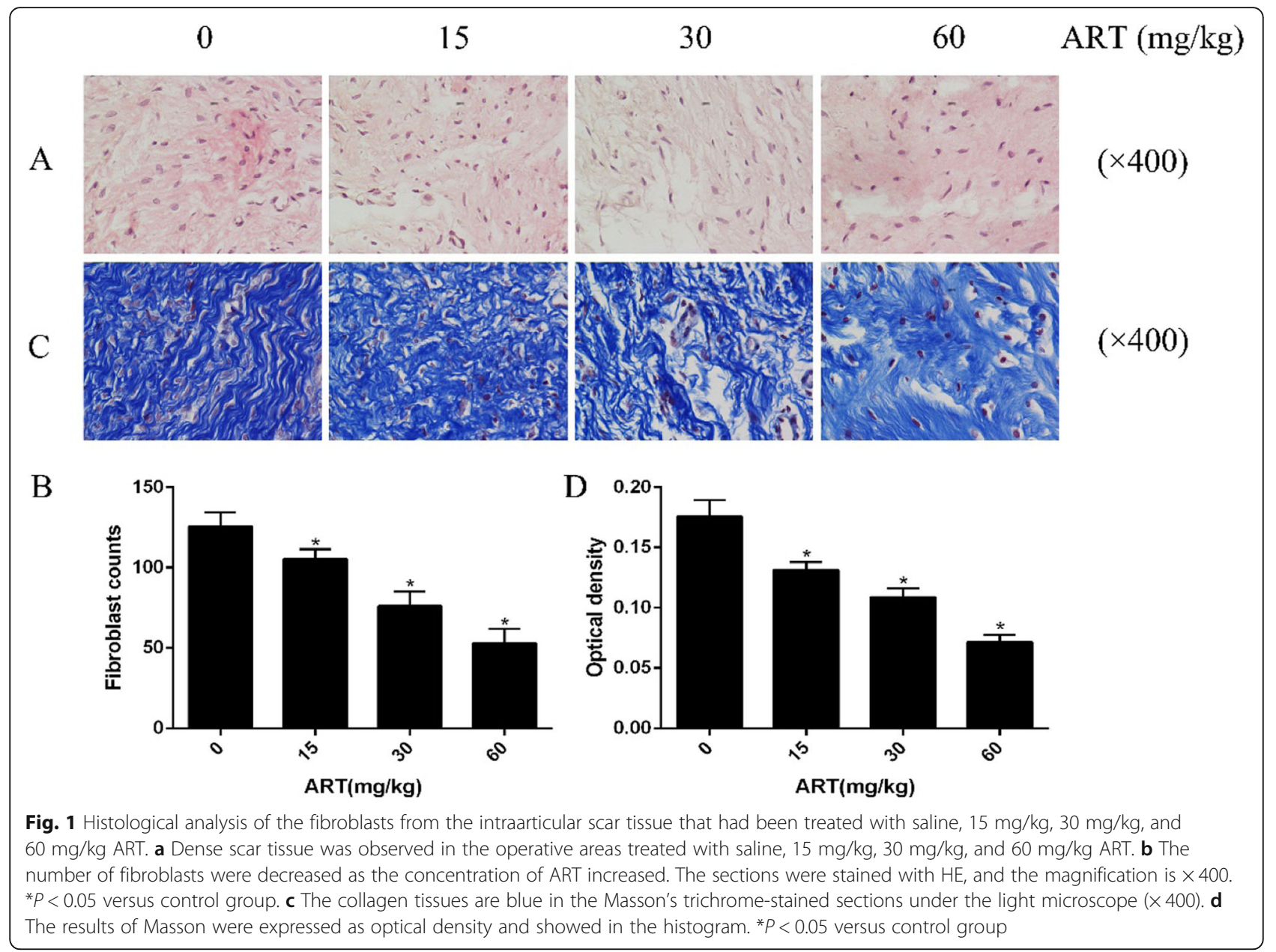

Bcl-2 expression was downregulated, and the ratio of Bax/Bcl-2 increased (Fig. 4a, b). Figure 4c, d showed that GRP78, the ratio of p-PERK/PERK and p-eIF $2 \alpha /$ eIF $2 \alpha$ also showed an increasing trend and the effect is concentration-dependent. On the other hand, we treated fibroblasts with ART at a concentration of $10 \mu \mathrm{M}$ for $0 \mathrm{~h}, 12 \mathrm{~h}, 24 \mathrm{~h}$, and $48 \mathrm{~h}$. The results showed that PERK pathway-related proteins were activated and gradually increased in a timedependent manner (Fig. 4e, f). To further investigate the role of PERK signal pathway in ART-induced fibroblast apoptosis, we use the PERK signal pathway inhibitor GSK2606414. The results showed that after the PERK pathway was inhibited, the expression of CHOP, GRP78, and Bax/Bcl-2 was decreased (Fig. 4g, h). CCK-8 results were similar (Fig. 4i); it was reported that after the inhibition of PERK pathway, the cell viability was higher than that of the normal drug treatment group. These results indicate that the ART could induce fibroblast apoptosis by activating the PERK signal pathway.

\section{Discussion}

ART is a derivative of artemisinin, which refers to a new structural antimalarial drug characterized by high efficiency, quick effect, low toxicity, and low tolerance. It has been adopted for mild to severe basic treatment for malaria infection. A growing number of studies have suggested that ART and its activated metabolite dihydroartemisinin exert pharmacological effects on antitumor, anti-inflammatory, anti-sepsis, anti-angiogenesis, anti-fibrosis, as well as immune regulation [9-12]. This study aimed to gain insights into the anti-fibrosis of ART.

Knee joint adhesion refers to a serious complication caused by knee surgery or knee injury. The clinical symptoms are largely limited by the range of knee joint activity and chronic joint pain; it eventually causes various sequelae (e.g., articular cartilage degeneration and knee joint stiffness), resulting in surgical failure. Existing studies considered that the major pathogenesis of knee adhesions includes [21, 22] (1) trauma/surgery caused early local soft tissue hemorrhage, activated inflammatory 


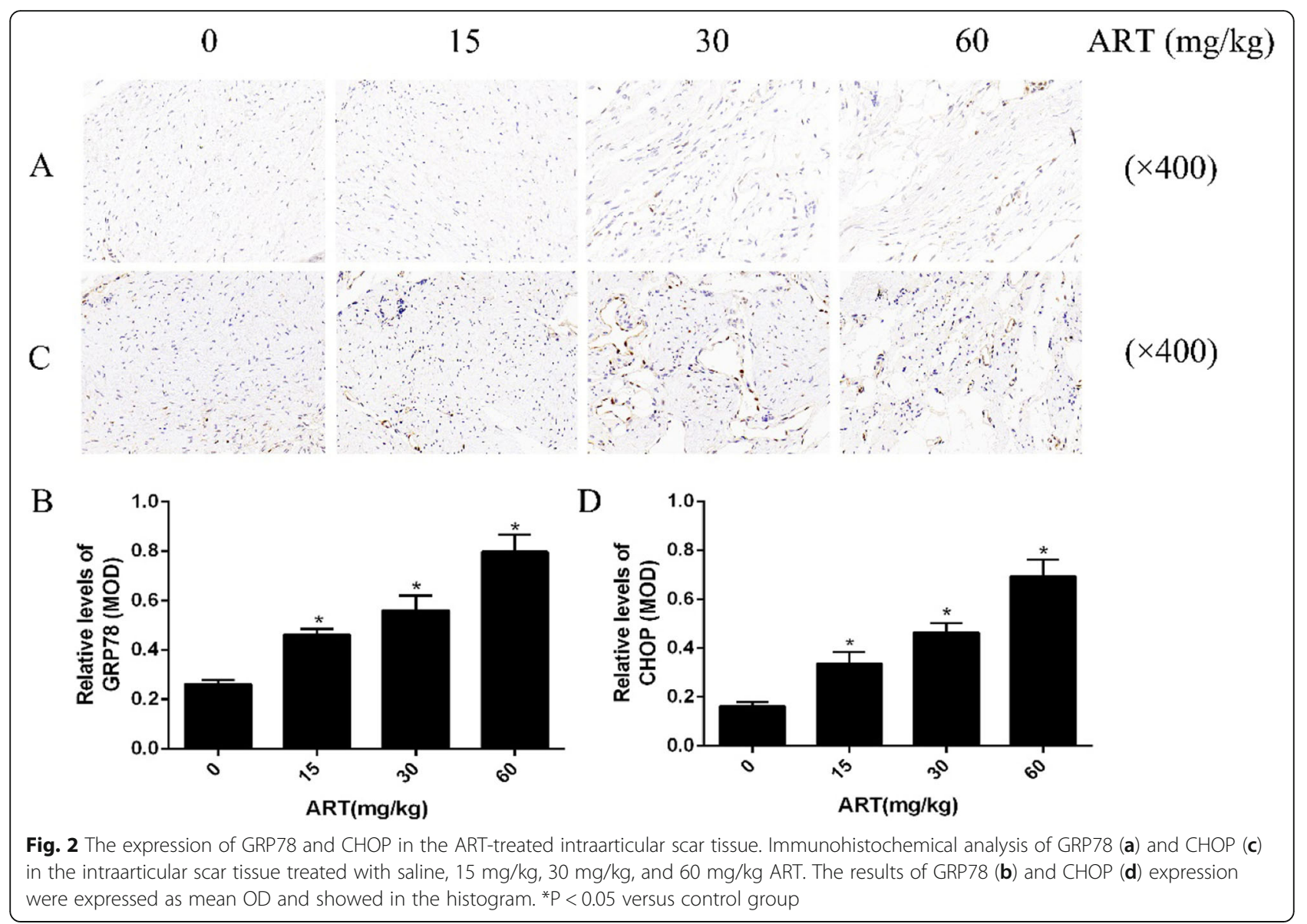

response, causing fibrin and inflammatory cell exudation; (2) fibrinolysis and the balance of the antifibrinolytic system are broken, causing fibrin deposition, and stimulating the migration, the proliferation, as well as the secretion of extracellular matrix (e.g., collagen and fibronectin), which eventually leads to the accumulation of granulation tissue around the synovial membrane and joint capsule; and (3) collagen fibers are thickened, and microvascular closure disappear, leading to in granulation tissue fibrosis, synovial membrane, and joint capsule atrophy, which results in the reduction of space in the joint cavity and the reduction of hyaluronic acid secretion, the formation of intraarticular adhesions, and joints degenerative lesions of cartilage.

This study was conducted to investigate the role and mechanism of ART-induced human fibroblasts in the prevention of intraarticular scar adhesion. In vivo experiments, HE staining results suggested that the fibroblast counts in the $60 \mathrm{mg} / \mathrm{kg}$ ART group were significantly lower than those in the ART low concentration group and the control group, and were drug concentration dependent. As the concentration of ART rises, the results of Masson staining and HE staining are consistent. Masson staining showed that the density of collagen fibers was lower in the $60 \mathrm{mg} / \mathrm{kg}$ ART group than in the low concentration group and the control group, and it was concentration-dependent. In vitro, ART-treated human fibroblasts and tested by cck- 8 showed that ART can reduce cell viability and promote fibroblast apoptosis in a concentration- and time-dependent manner. On the other hand, results of tunnel staining, flow cytometry, and increase of cleaved-PARP expression in ART-treated fibroblasts also indicates the apoptosis of fibroblasts. The above experiments in vitro and in vivo showed that ART can promote the apoptosis of fibroblasts and reduce the production of collagen fibers.

ER is a multifunctional organelle that plays an important role in maintaining the stability of the intracellular environment. After ERS occurs, the cells initiate an UPR to restore the homeostasis of the cells [23]. Under longterm severe ERS, UPR no longer exerted cytoprotective effects but started to exert cytotoxic effects (including apoptosis). Some scholars reported that the use of ingenuity pathway analysis (IPA) found that UPR is a crucial way to induce ERS by ART [24]. The PERK 


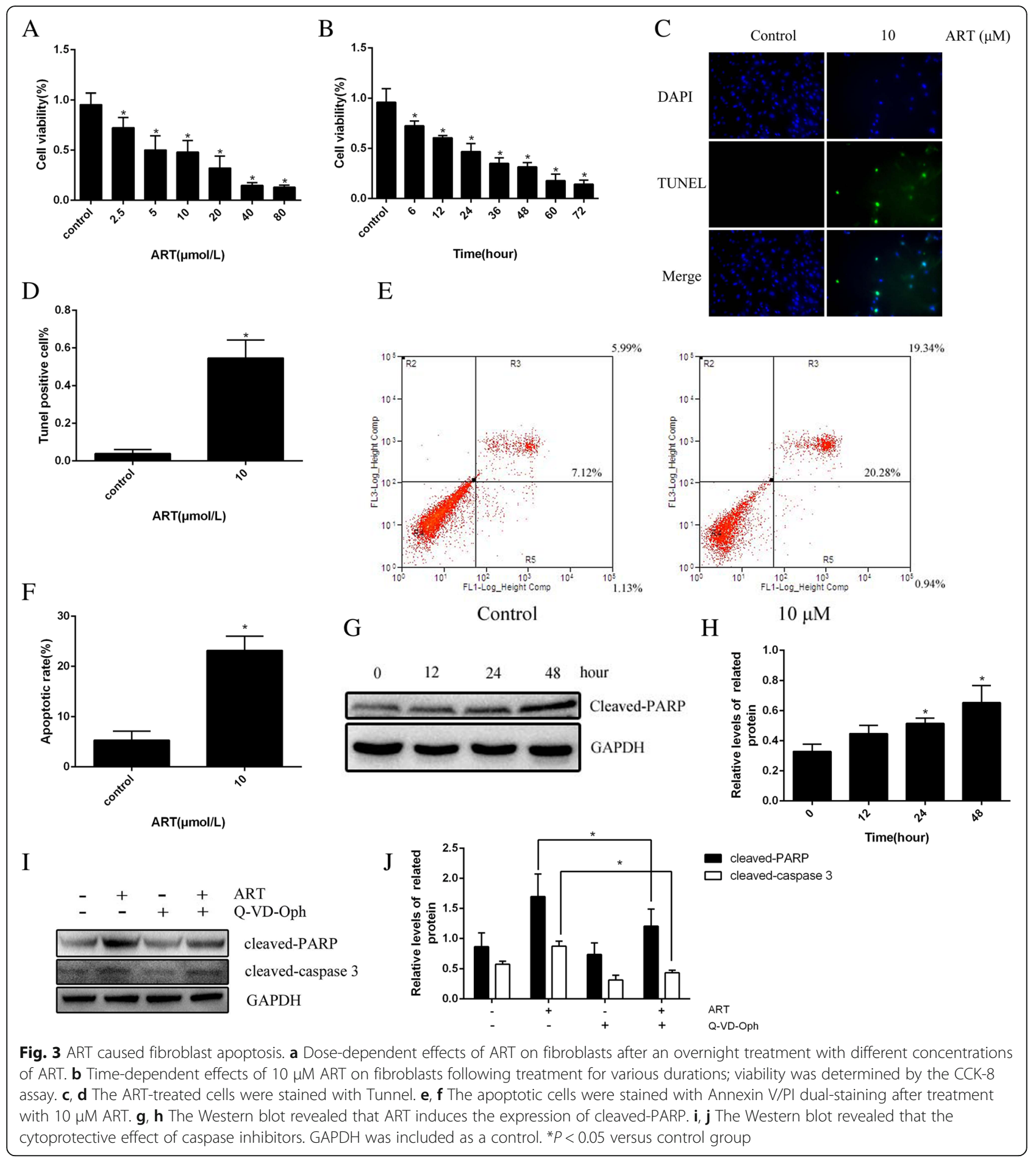

pathway is one of the classic ERS pathways. In the present study, upregulated expression of GRP78, CHOP, and Bax in fibroblasts after ART treatment, and the expression of $\mathrm{Bcl}-2$ was downregulated, suggesting that ERS is activated. Furthermore, after ART treatment, the proteins (e.g., p-PERK and p-eIF2 $\alpha$ increased), and the ratios of p-PERK to PERK and p-eIF2 $\alpha$ to eIF2 $\alpha$ in the PERK pathway were also upregulated, which was also concentration- and time-dependent, suggesting that the PERK pathway is involved in ART-mediated apoptosis. Furthermore, after treatment with GSK2606414, we found that the increasing trend of CHOP, GRP78, and 
A

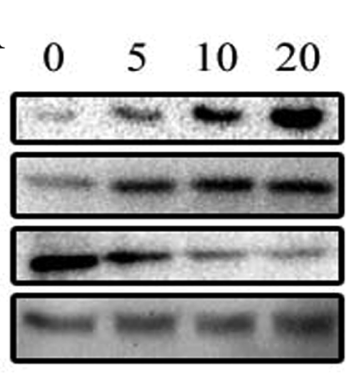

C

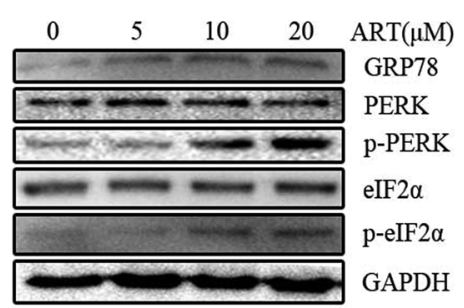

E



G

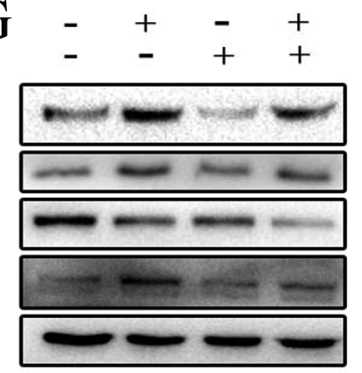

ART

GRP78

CHOP

Bcl-2

$\mathrm{Bax}$

GAPDH

ART $(\mu \mathrm{M})$
CHOP
Bax
Bcl-2
GAPDH

D
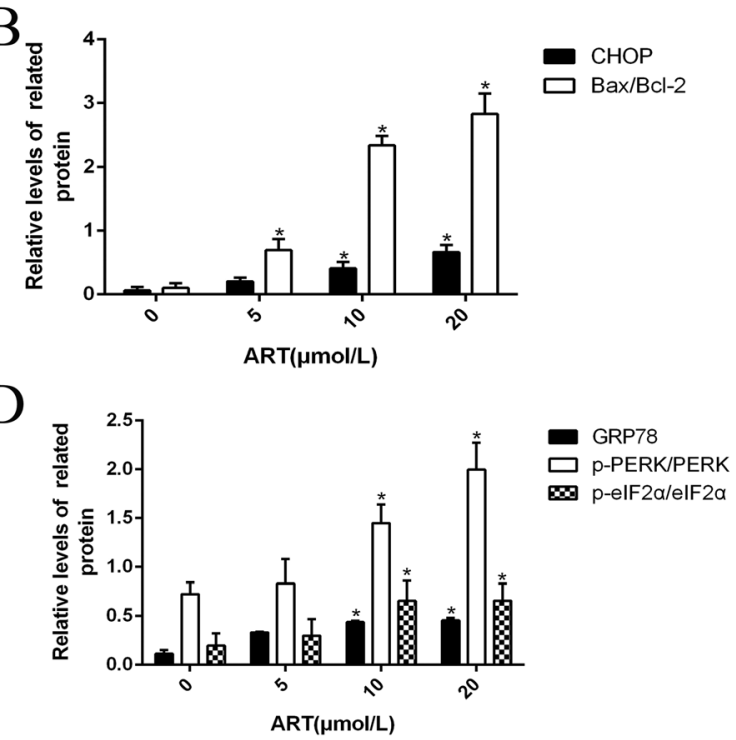

F

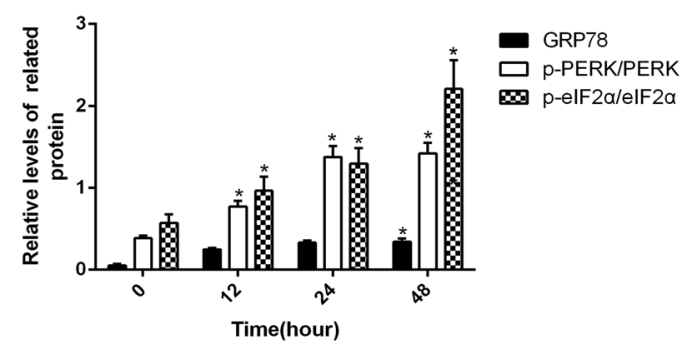

$\mathrm{H}$

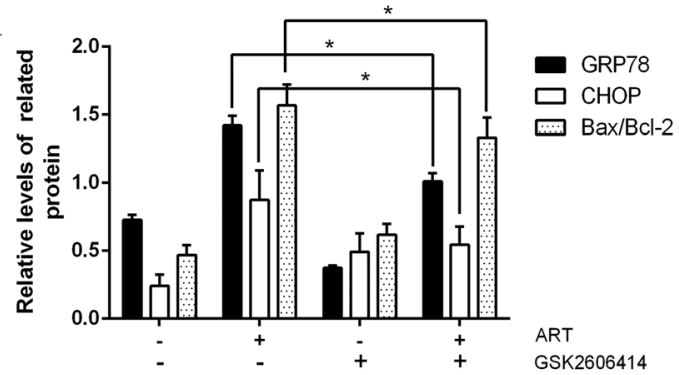

GSK2606414

GSK2606414

I

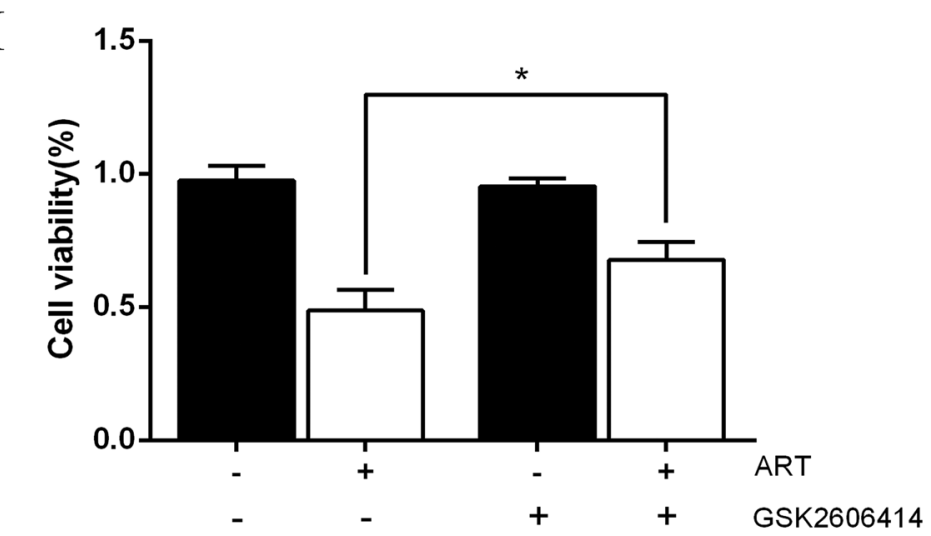

Fig. 4 (See legend on next page.) 
(See figure on previous page.)

Fig. 4 ART induced ER stress in the fibroblasts. $\mathbf{a}$, b The expression of CHOP, BCl-2, and Bax in cells that had been treated with $0,5 \mu \mathrm{M}$, $10 \mu \mathrm{M}$, 20 MM ART overnight was determined by western blot analysis. GAPDH was included as a control. c-f The dose- and time-dependent effects of ART on the expression of proteins associated with the PERK signaling pathway, including p-PERK, PERK, p-elF2a, and elF2a, were determined by western blot analysis. GAPDH was used as a control. $\mathbf{g}$, $\mathbf{h}$ After treated with or without GSK2606414, the fibroblasts were treated with $10 \mu \mathrm{MM}$ ART for $24 \mathrm{~h}$, the detected by Western blotting with antibodies specific for GRP78, CHOP, Bax, Bcl-2, and GAPDH. i After treated with or without GSK2606414, the fibroblasts were treated with ART as described before and then cell viability was measured. ${ }^{*} P<0.05$ versus control group

Bax/bcl-2 was inhibited. Therefore, these results indicate that ART induces fibroblast apoptosis may be through the PERK pathway involved in the process of apoptosis.

\section{Conclusion}

In conclusion, this preliminary study revealed that topical injection of ART could prevent joint scar adhesion. The possible mechanism may be the apoptosis of fibroblasts induced by PERK signaling pathway. Nevertheless, more experiments are required to verify the safety, efficacy, and toxicity of ART.

\begin{abstract}
Abbreviations
ART: Artesunate; CCK-8: Cell counting kit-8; CHOP: C/EBP homologous protein; DMEM: Dulbecco's modified Eagle's medium; elF2a: Eukaryotic initiation factor 2a; ERS: Endoplasmic reticulum stress; FBS: Fetal bovine serum; GAPDH: Glyceraldehyde-3-phosphate dehydrogenase; GRP78: 78 kDa glucose-regulated protein 78; HE: Hematoxylin-eosin; PARP: Poly ADP-ribose polymerase; PBS: Phosphate buffer saline; PERK: PRKR-like ER kinase; PVDF: Polyvinylidene difluoride; RIPA: Radioimmune-deposition; SDSPAGE: Sodium dodecyl sulphate-polyacrylamide gel electrophoresis; TBST: Tris-buffered saline and Tween20; UPR: Unfolded protein response
\end{abstract}

\section{Acknowledgments}

We show great appreciation to all of our teachers for their generous help.

\section{Authors' contributions}

$\mathrm{HC}$ and JT performed the whole experiments and were responsible for the data and drafting the article. JW and LY designed the study and contributed to the preparation of the manuscript. All authors read and approved the final manuscript.

\section{Funding}

This study was supported by the National Natural Science Foundation of China (Grant no. 81772332). This study was also supported by Jiangsu Provincial Medical Innovation Team (Grants\#CXTDB2017004).

\section{Availability of data and materials}

The datasets supporting the conclusions of this article are included within the article and its supplementary materials.

\section{Ethics approval}

This experimental study was approved by the Ethics Committee and Research Committee of Jiangsu Subei People's Hospital. The animal experiment was approved by the Animal Ethics Committee of Yangzhou University, and all the rabbits received humanitarian care.

\section{Consent for publication}

Not applicable.

\section{Competing interests}

We declare that we have no financial and personal relationships with other people or organizations that can inappropriately influence our work. There is no professional or other personal interest of any nature or kind in any product, service, and/or company that could be construed as influencing the position presented in, or the review of, the manuscript entitled.

\section{Author details}

'Dalian Medical University, Dalian, Liaoning, People's Republic of China. ${ }^{2}$ Department of Orthopedics, Clinical Medical College of Yangzhou University, Subei People's Hospital of Jiangsu Province, Yangzhou, People's Republic of China.

Received: 30 July 2019 Accepted: 31 October 2019

Published online: 17 December 2019

\section{References}

1. Bieger R, Kappe T, Fraitzl CR, Reichel H. The aetiology of total knee arthroplasty failure influences the improvement in knee function. Arch Orthop Traum Su. 2013;133:237-41.

2. Issa K, Banerjee $\mathrm{S}$, Kester MA, et al. The effect of timing of manipulation under anesthesia to improve range of motion and functional outcomes following Total knee Arthroplasty. J Bone Joint Surg. 2014;96:1349-57.

3. Wang M, Liu C, Xiao W. Intra-articular injection of hyaluronic acid for the reduction in joint adhesion formation in a rabbit model of knee injury. Knee Surg Sports Traumatol Arthrosc. 2014;22:1536-40.

4. Sandoval MA, Hernandez-Vaquero D. Preventing peridural fibrosis with nonsteroidal anti-inflammatory drugs. Eur Spine J. 2008;17:451-5.

5. Kocaoglu B, Akgun U, Nalbantoglu U, Poyanli O, Karahan M. Adhesion reduction after knee surgery in a rat model by mitomycin C. Knee Surg Sports Traumatol Arthrosc. 2011;19:94-8.

6. Wang J, Yan L, Sun Y, et al. A comparative study of the preventive effects of mitomycin $\mathrm{C}$ and chitosan on intraarticular adhesion after knee surgery in rabbits. Cell Biochem Biophys. 2012;62:101-5.

7. Bar-Zeev N, White N. Evidence behind the WHO guidelines: hospital care for children: efficacy and safety of artemisinin derivatives in children with malaria. J Trop Pediatr. 2006;52:78-82.

8. Ribeiro IR, Olliaro P. Safety of artemisinin and its derivatives. A review of published and unpublished clinical trials. Médecine tropicale: revue du Corps de santé colonial. 1998:58(3 Suppl):50-3.

9. Jiang $W, C e n ~ Y$, Song $Y$, et al. Artesunate attenuated progression of atherosclerosis lesion formation alone or combined with rosuvastatin through inhibition of pro-inflammatory cytokines and pro-inflammatory chemokines. Phytomedicine. 2016:23:1259-66.

10. Cen Y, Liu C, Li X, et al. Artesunate ameliorates severe acute pancreatitis (SAP) in rats by inhibiting expression of pro-inflammatory cytokines and toll-like receptor 4. Int Immunopharmacol. 2016;38:252-60.

11. Zhao $Y$, Jiang $W$, Li B, et al. Artesunate enhances radiosensitivity of human non-small cell lung cancer A549 cells via increasing NO production to induce cell cycle arrest at G2/M phase. Int Immunopharmacol. 2011;11: 2039-46.

12. Li B, Li J, Pan X, et al. Artesunate protects sepsis model mice challenged with Staphylococcus aureus by decreasing TNF-alpha release via inhibition TLR2 and Nod2 mRNA expressions and transcription factor NF-kappaB activation. Int Immunopharmacol. 2010;10:344-50.

13. Lai $L$, Chen $Y$, Tian $X$, et al. Artesunate alleviates hepatic fibrosis induced by multiple pathogenic factors and inflammation through the inhibition of LPS/TLR4/NF-kappaB signaling pathway in rats. Eur J Pharmacol. 2015;765:234-41.

14. Liu Y, Huang G, Mo B, Wang C. Artesunate ameliorates lung fibrosis via inhibiting the notch signaling pathway. Exp Ther Med. 2017;14:561-6.

15. Cao J, Wang W, Li Y, et al. Artesunate attenuates unilateral ureteral obstruction-induced renal fibrosis by regulating the expressions of bone morphogenetic protein-7 and uterine sensitization-associated gene-1 in rats. Int Urol Nephrol. 2016;48:619-29.

16. Du Y, Li LN, Fang BW. Effects of Artesunate on hepatic fibrosis and its mechanism. Zhongguo Ying Yong Sheng Li Xue Za Zhi. 2015;31(1):14-7. 
17. Ferri KF, Kroemer G. Organelle-specific initiation of cell death pathways. Nat Cell Biol. 2001;3:E255-63.

18. Zinszner $\mathrm{H}$, Kuroda $\mathrm{M}$, Wang $\mathrm{X}$, et al. CHOP is implicated in programmed cell death in response to impaired function of the endoplasmic reticulum. Genes Dev. 1998;12:982-95.

19. Shi K, Wang D, Cao X, Ge Y. Endoplasmic reticulum stress signaling is involved in mitomycin C (MMC)-induced apoptosis in human fibroblasts via PERK pathway. PLoS One. 2013;8:e59330.

20. Liang Y, Sun Y, Li X, et al. The optimal concentration of topical hydroxycamptothecin in preventing intraarticular scar adhesion. Sci Rep. 2014:4:4621.

21. Mercer PF, Chambers RC. Coagulation and coagulation signalling in fibrosis. Biochim Biophys Acta. 1832;2013:1018-27.

22. Lieber RL, Ward SR. Cellular mechanisms of tissue fibrosis. 4. Structural and functional consequences of skeletal muscle fibrosis. Am J Physiol Cell Physiol. 2013;305:C241-52.

23. Wan S, Jiang L. Endoplasmic reticulum (ER) stress and the unfolded protein response (UPR) in plants. Protoplasma. 2016;253(3):753-64.

24. Vătsveen TK, Myhre MR, Steen CB, et al. Artesunate shows potent antitumor activity in B-cell lymphoma. J Hematol Oncol. 2018;11(1):23.

\section{Publisher's Note}

Springer Nature remains neutral with regard to jurisdictional claims in published maps and institutional affiliations.

Ready to submit your research? Choose BMC and benefit from:

- fast, convenient online submission

- thorough peer review by experienced researchers in your field

- rapid publication on acceptance

- support for research data, including large and complex data types

- gold Open Access which fosters wider collaboration and increased citations

- maximum visibility for your research: over $100 \mathrm{M}$ website views per year

At $\mathrm{BMC}$, research is always in progress.

Learn more biomedcentral.com/submissions 